\title{
Clinical Utility of Short Social Cognitive Tests in Early Differentiation of Behavioral Variant Frontotemporal Dementia from Alzheimer's Disease
}

\author{
Christian Buhl $^{a}$ Jette Stokholm ${ }^{a}$ Anders Gade $^{b}$ \\ a Memory Disorders Research Group, Danish Dementia Research Center, Department of Neurology, \\ Copenhagen University Hospital, Rigshospitalet, and bepartment of Psychology, University of \\ Copenhagen, Copenhagen, Denmark
}

\section{Key Words}

Frontotemporal dementia - Alzheimer's disease · Social cognition · The Awareness of Social Inference Test · Reading the Mind in the Eyes Test - Emotion Hexagon

\begin{abstract}
Background: Traditional cognitive tests used in clinical practice may not be sensitive enough for the early differentiation of behavioral variant frontotemporal dementia (bvFTD) from Alzheimer's disease (AD). A growing body of literature has shown that deficits in various aspects of social cognition can be found in bvFTD. Aim: The objective of this study is to investigate whether short and easily administered tests of social cognition are useful in providing clinical information which might aid in the differentiation of bvFTD from AD in the early stages of bvFTD. Methods: 11 patients diagnosed with bvFTD and 10 patients diagnosed with AD completed a neuropsychological assessment comprising global, executive and social cognitive tasks. Results: Measures of global cognitive function showed no significant difference between the two groups, whereas even the short social cognitive measures (the Reading the Mind in the Eyes Test and the Emotion Hexagon) showed significant group differences, reflecting a poorer performance by the bvFTD group. Conclusion: Our results suggest that it may indeed be relevant to include short and easily administered measures of social cognition in the differential diagnosis of early bvFTD and AD.

(C) 2013 S. Karger AG, Basel
\end{abstract}

The Awareness of Social Inference Test (TASIT): Copyright ${ }^{\circledR} 2002$ by Skye McDonald, Sharon Flanagan and Jennifer Rollins, published by Pearson, Clinical Assessment; Danish Copyright ${ }^{\circledR} 2013$ by Pearson, Clinical Assessment. Adapted and reproduced with permission. All rights reserved. 


\section{Introduction}

While the description of the clinical core symptoms of behavioral variant frontotemporal dementia (bvFTD) [1] and Alzheimer's disease (AD) [2] suggests that they represent quite distinct and easily discriminable syndromes, most clinicians will agree that this is often not the case in clinical practice. Also, studies have demonstrated that the cognitive tests typically used for dementia evaluation may not be able to discriminate between these diseases $[3,4]$.

Patients with bvFTD typically present with insidious changes in social conduct and personality [1,5] and exhibit behavioral problems that suggest executive dysfunction. However, this is not always reflected in their performance on executive tests [6-9]. Other bvFTD patients show deficits on executive tests, but they also do so on tests of other cognitive functions such as memory, and they can therefore resemble AD patients in their cognitive profile $[10,11]$.

In recent years, a growing body of literature has shown that deficits in various aspects of social cognition, e.g. theory of mind, empathy, emotion recognition or discriminating sincere from sarcastic exchanges, can be found in bvFTD $[6,12-20]$. This is in agreement with reported atrophy of the orbital and medial prefrontal regions [21,22], and several studies have indicated a link between these brain areas and social cognitive processes in the early stages of bvFTD [22-24]. Furthermore, various social cognitive tasks have also shown promising results in their ability to differentiate patients with bvFTD from patients with AD [15, 17-19]. However, many of the tests used in these studies are time consuming and not suitable for clinical practice. It is therefore of great importance to develop clinically applicable instruments that are sensitive to the early and specific deficits seen in patients with bvFTD [25], and promising results have already been shown with more clinically applicable tools that assess social cognition such as the Social Cognition and Emotional Assessment (SEA) [19] and its abbreviated version, the Mini-SEA [26].

The aim of this study was therefore to further investigate whether short and easily administered tests of social cognition are useful in providing clinical information which might aid in the differentiation of bvFTD from AD in the early stages of bvFTD.

\section{Methods}

\section{Subjects}

In this study we included a total of 21 patients, 11 patients diagnosed with bvFTD (7 males and 4 females) and 10 patients diagnosed with AD ( 9 males and 1 female). Patients were recruited from the Copenhagen University Hospital Memory Clinic, which is an outpatient clinic based in a neurological setting. All patients had undergone an extensive clinical assessment program, including a neurological examination, neuropsychological assessment, laboratory screening, electrocardiography and structural neuroimaging with either computed tomography or magnetic resonance imaging. Most patients had also undergone functional neuroimaging using positron emission tomography or single-photon emission computed tomography. All bvFTD patients had presented with a corroborated history of initial progressive change in personality and behavior and fulfilled the international consensus criteria for bvFTD [1]. All AD patients fulfilled the NINCDS-ADRDA criteria for probable AD [27].

The bvFTD patients were drawn from the Copenhagen Memory Clinic patient database. In order to identify patients at an early stage of their disease, only those who had been diagnosed with bvFTD no more than 3 years prior to the study and had a Mini-Mental State Exam- 
Buhl et al.: Clinical Utility of Short Social Cognitive Tests in Early Differentiation of Behavioral Variant Frontotemporal Dementia from Alzheimer's Disease

ination (MMSE) score of 25 and above were included. Exclusion criteria were moderate or severe depression, alcohol or drug abuse, major head injury or previous cerebrovascular episodes. Patients living too far from our clinic or presenting with a significant degree of semantic impairment that might interfere with their comprehension of the tasks were also excluded. The $10 \mathrm{AD}$ patients were specifically picked from the database in order to match the patients in the bvFTD group with respect to age, educational level and MMSE.

\section{Procedure}

All subjects completed a neuropsychological assessment comprising global, executive and social cognitive tasks in one session lasting about 1.5-2 h. Video vignettes were presented on a 13-inch Acer laptop computer. Education index scores were calculated as the sum of years at school (7-12) and a level of education index (range 1-5) [28].

Global Cognitive Tasks

As a general assessment of global cognitive function, subjects were given the MMSE [29] and the Addenbrooke's Cognitive Examination (ACE) [30, 31].

\section{Executive Tasks}

The Frontal Assessment Battery (FAB) [32] is a short cognitive and behavioral battery to assess frontal lobe functions and consists of six subtests: conceptualization, mental flexibility, motor programming, sensitivity to interference, inhibitory control and environmental autonomy. A score of $0-3$ is possible in each subtest yielding a maximum total of 18 points. A low score indicates executive impairment.

The Hayling task [33] is a sentence completion task that tests inhibition of an automatic response. In two separate sections, subjects have to complete sentences with either an expected or unrelated word. Errors and response times result in an overall score, which is transformed to an overall scaled score with a maximum of 10 .

The Brixton Spatial Anticipation Task [33] is a spatial rule detection task, testing the ability to detect and adapt to rules in a series of stimuli. The total number of errors as well as a converted scaled score with a maximum of 10 was used to measure outcome.

\section{Social Cognitive Tasks}

The Emotion Hexagon is a test where subjects must correctly identify the dominant emotion in different facial images. We used a modified version of the Emotion Hexagon [34]. The subjects had to judge a total of 30 images depicting a variety of 'morphed' facial expressions for which one and the same model had posed. Each image consisted of two 'morphed' facial expressions of emotion. The images were printed on cards $5 \times 7 \mathrm{~cm}$ in size and were presented one at a time in random order and only once. The subjects had to choose one of six possible emotions for each facial expression: anger, happiness, disgust, surprise, sadness and fear, with five images related to each emotion. The six possible emotions were printed on a card which the subjects were free to consult throughout the test. When the image was a 50/50 mix between two emotions, the item was not scored. All other combinations were scored, leading to a maximum score of 24 correct items. The Emotion Hexagon takes approximately 5-10 min to administer.

The Reading the Mind in the Eyes Test revised version (RME) [35] is a theory of mind test consisting of photographs of the eye region of 36 faces. With each picture the subjects are presented with four mental state words and have to choose the word that best describes what the person in the picture is thinking or feeling. If the subjects are unsure of a word, a glossary is provided. The test takes approximately $10-15 \mathrm{~min}$ to administer and has a maximum of 36 correct answers. 
Buhl et al.: Clinical Utility of Short Social Cognitive Tests in Early Differentiation of Behavioral Variant Frontotemporal Dementia from Alzheimer's Disease

The Emotion Evaluation Task (EET) (form A) is a subtest of The Awareness of Social Inference Test (TASIT) [36]. The EET examines the subject's ability to identify six basic emotions (happiness, surprise, sadness, anger, fear and disgust) or no particular emotion (neutral) in 28 professionally enacted short video vignettes (15-60 s). Since the original version contains English language, our subjects were shown the vignettes without sound to avoid a language bias. Even though our subjects did not have sound or intonation available, the EET is still thought to be a more ecologically valid test of emotion recognition than mere static stimuli, because it is dynamic and incorporates naturalistic expressions and gestural cues. In scenes containing two actors, the subjects were instructed to focus on one of them. After having viewed each scene, the subjects were asked to state the emotion portrayed by the actor with the help of a response card where the emotions are given in random order. The subjects were allowed to watch each vignette as many times as they wanted in order to exclude a memory bias. Scores were summed in positive emotions (happiness, surprise and neutral) with a maximum score of 12 and negative emotions (sadness, anger, fear and disgust) with a maximum score of 16 . Scores for positive and negative emotions were also added to a total maximum score of 28. The EET takes roughly 30-40 min to administer.

The Social Inference-Minimal Test (SI-M) is a subtest of TASIT [36]. We used a Danish version of the SI-M to examine understanding of conversational meanings which are determined by paralinguistic cues (facial expression, intonation, gesture, etc.), since the dialogue is neutral in content. The SI-M consists of 15 video vignettes with two actors portraying everyday conversational interactions in which the actors make sincere, sarcastic or paradoxical conversational remarks. In the sincere vignettes, the dialogue and the context are consistent, and hence the actors mean what they say. In the sarcastic vignettes, one of the actors actually means the opposite of what he says, and since the dialogue is neutral in content, this is conveyed by paralinguistic cues such as facial expression or tone of voice. In the paradoxical vignettes, the dialogue makes no sense, unless it is recognized that one of the actors is sarcastic. A failure to detect the paralinguistic cues will lead the subject to misinterpret these vignettes. After each vignette, comprehension was assessed by four yes-no questions regarding what the actor had done, said, felt and thought. Again subjects were allowed to watch each vignette as many times as they wanted in order to exclude a memory bias. A score was calculated for each type of conversational remark (sincere, sarcastic or paradoxical) with a maximum score of 20 . A total score with a maximum of 40 was also summed for both types of sarcastic remarks (total sarcastic). A total score was calculated for all conversational remarks, with a maximum score of 60 . The SI-M can be administered in approximately 20 $25 \mathrm{~min}$.

\section{Statistical Analysis}

Statistical analysis was performed using SPSS version 19.0. Exploration of the data using the Kolmogorov-Smirnov test revealed that the data was not normally distributed. In view of the relatively small group sizes and the nonnormal distribution of our data, nonparametric statistics were applied. Mann-Whitney U tests were used to analyze the group differences, and scores are reported as median values and range. Pearson correlations were used for correlation analyses. Logistic regression analysis was performed to assess the impact of the ability of the social cognitive tests to predict the diagnostic group, and receiver operating characteristics (ROC) curves were applied too in order to evaluate the discriminating power of the social cognitive tests compared to the executive tests (FAB, Hayling and Brixton). The area under the curve (AUC) was used to measure the overall performance of each ROC curve. 
Table 1. Background data as well as global cognitive and executive tasks

Table 2. Social cognitive tasks

\begin{tabular}{l|l}
\hline \multicolumn{2}{l}{ Dement Geriatr Cogn Disord Extra 2013;3:376-385 } \\
\hline DOI: $10.1159 / 000355123$ & $\begin{array}{l}\text { C 2013 S. Karger AG, Basel } \\
\text { www.karger.com/dee }\end{array}$ \\
\hline
\end{tabular}

Buhl et al.: Clinical Utility of Short Social Cognitive Tests in Early Differentiation of Behavioral Variant Frontotemporal Dementia from Alzheimer's Disease

\begin{tabular}{lcrl}
\hline Variable & bvFTD $(\mathrm{n}=11)$ & $\mathrm{AD}(\mathrm{n}=10)$ & $\mathrm{p}$ value \\
\hline Age & $67(54-76)$ & $66(51-72)$ & 0.931 \\
Education & $16.0(11-17)$ & $14.5(11-17)$ & 0.686 \\
MMSE & $28(25-30)$ & $28(25-30)$ & 0.615 \\
ACE & $83(71-91)$ & $84.5(77-90)$ & 0.545 \\
FAB & $14(8-18)$ & $17(12-18)$ & 0.141 \\
Brixton task (total errors) $^{1}$ & $28(16-48)$ & $27(18-50)$ & 0.959 \\
Hayling task (scaled score) & $2(1-3)$ & $3(1-6)$ & $0.042^{*}$ \\
\hline
\end{tabular}

Values represent median (range). Age is reported in years. Test performance scores are either reported as raw scores or scaled scores. ${ }^{*} \mathrm{p}<0.05 .{ }^{1}$ Higher scores indicate worse performance.

\begin{tabular}{lccc}
\hline Variable & bvFTD $(\mathrm{n}=11)$ & $\mathrm{AD}(\mathrm{n}=10)$ & $\mathrm{p}$ value \\
\hline Emotion Hexagon & $14(9-20)$ & $18(15-19)$ & $0.022^{*}$ \\
RME & $15(6-24)$ & $21.5(19-26)$ & $0.004^{*}$ \\
EET & & \\
$\quad$ Positive emotions & $9(5-10)$ & $9(7-11)$ & 0.619 \\
$\quad$ Negative emotions & $7(4-12)$ & $10(4-13)$ & $0.026^{*}$ \\
$\quad$ Total emotions & $16(10-21)$ & $19(15-22)$ & $0.022^{*}$ \\
SI-M & & & \\
$\quad$ Sincere & $14(5-18)$ & $12.5(9-19)$ & 0.393 \\
$\quad$ Sarcastic & $13(5-17)$ & $16(13-20)$ & $0.005^{*}$ \\
$\quad$ Paradoxical sarcastic & $15(7-20)$ & $19(16-20)$ & $0.002^{*}$ \\
$\quad$ Total sarcastic & $28(12-34)$ & $35(32-39)$ & $0.000^{*}$ \\
$\quad$ Total & $41(27-48)$ & $47.5(44-52)$ & $0.002^{*}$ \\
\hline
\end{tabular}

Values represent median (range). Test performance scores are reported as raw scores. ${ }^{*} \mathrm{p}<0.05$.

\section{Results}

\section{Global Cognitive Function and Executive Tests}

Results of the global cognitive tests and executive tests are presented together with demographic data in table 1. Comparing the measures of global cognitive function showed no significant difference between the two groups. Among the executive tasks only the Hayling task showed a significant group difference $(p=0.042)$. On this task the bvFTD group had a significantly lower scaled score, reflecting poorer performance than the AD group. There was a trend towards significance on the FAB $(p=0.141)$ with the bvFTD group scoring lower than the AD group.

\section{Social Cognitive Tests}

Results of the social cognitive tests are presented in table 2 . There was a significant group difference on the Emotion Hexagon ( $p=0.022)$ and the RME ( $=0.004)$, in which the bvFTD group had significantly lower performance scores than the AD group. On the EET, the bvFTD group had a significantly poorer performance than the AD group on scores for negative emotions $(p=0.026)$ and total emotions $(p=0.022)$. On the SI-M, the bvFTD group showed a significantly poorer performance than the AD group on measures of sarcastic $(p=0.005)$, para- 
Dementia

and Geriatric

Table 3. Correlation between scores on the social cognitive tests

Fig. 1. ROC curves for the four main social cognitive tests (RME, Emotion Hexagon, EET and SI-M) compared with the three executive measures (FAB, Hayling and Brixton).

\begin{tabular}{l|l}
\hline Dement Geriatr Cogn Disord Extra 2013;3:376-385 \\
\hline DOI: $10.1159 / 000355123$ & $\begin{array}{l}\text { @ 2013 S. Karger AG, Basel } \\
\text { www.karger.com/dee }\end{array}$ \\
\hline
\end{tabular}

Buhl et al.: Clinical Utility of Short Social Cognitive Tests in Early Differentiation of Behavioral Variant Frontotemporal Dementia from Alzheimer's Disease

\begin{tabular}{llll}
\hline & $\begin{array}{l}\text { Emotional } \\
\text { Hexagon }\end{array}$ & RME & EET \\
\hline RME & $0.65^{* *}$ & - & \\
EET & $0.59^{* *}$ & $0.57^{* *}$ & - \\
SI-M & $0.52^{*}$ & $0.78^{* *}$ & $0.66^{* *}$ \\
\hline
\end{tabular}

${ }^{*} \mathrm{p}<0.05 ; * * \mathrm{p}<0.01$

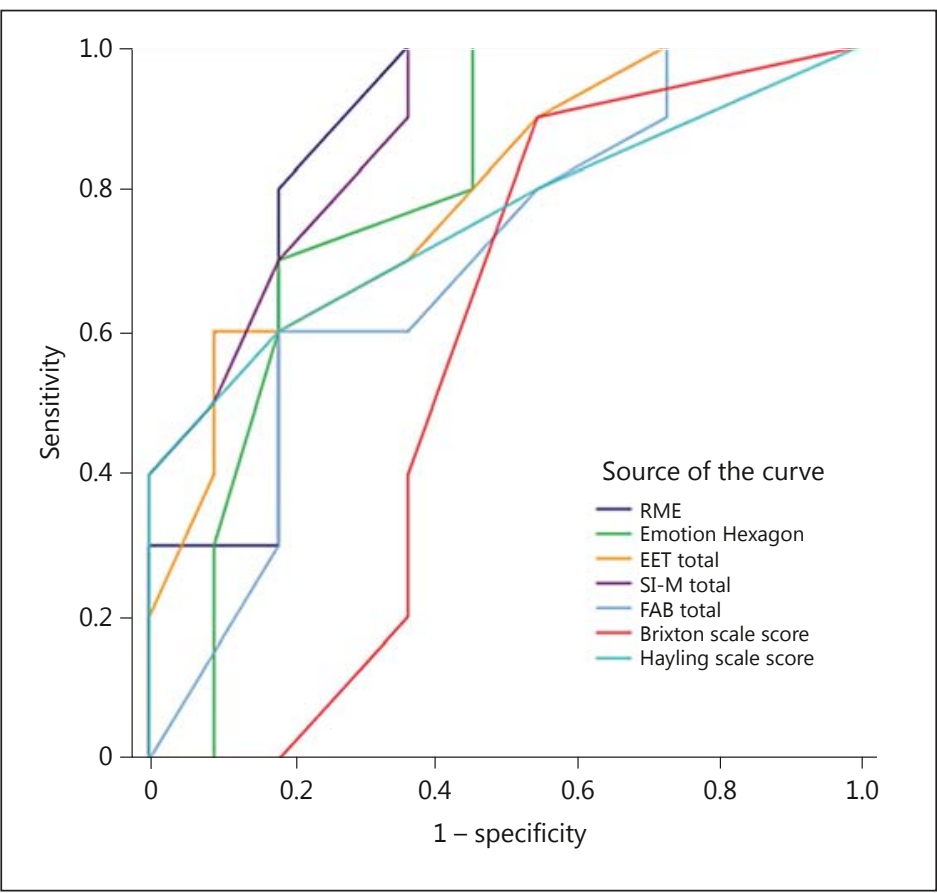

doxical sarcastic $(p=0.002)$, total sarcastic $(p=0.000)$ and total $(p=0.002)$ remarks. There was no significant group difference between the two patient groups on the SI-M sincere score.

\section{The Clinical Value of the Social Cognitive Tests}

There were significant and positive associations between scores on the four main social cognitive measures, with most correlation coefficients between 0.5 and 0.7 . The strongest correlation was between RME and SI-M ( $r=0.78)$, suggesting that these two tests measure almost the same aspects of social cognition (table 3 ).

Logistic regression was performed in order to assess the impact of each of the social cognitive tests on the diagnostic classification of the patients. The RME score explained between $36.2 \%$ (Cox and Snell $\mathrm{R}^{2}$ ) and $48.3 \%$ (Nagelkerke $\mathrm{R}^{2}$ ) and correctly classified $81 \%$ of the patients. The SI-M score explained between 44.9\% (Cox and Snell $\mathrm{R}^{2}$ ) and 59.9\% (Nagelkerke $\mathrm{R}^{2}$ ) and correctly classified $76 \%$ of the patients. The score on the Emotion Hexagon explained between 28.7\% (Cox and Snell $\mathrm{R}^{2}$ ) and $38.2 \%$ (Nagelkerke $\mathrm{R}^{2}$ ) and correctly classified $71 \%$ of the patients, and the EET score explained between $26.4 \%$ (Cox and Snell $\mathrm{R}^{2}$ ) and $35.2 \%$ (Nagelkerke $\mathrm{R}^{2}$ ) and also correctly classified $71 \%$ of the patients.

There was no added benefit to be gained by combining two or more social cognitive tests in the logistic regression model. The ROC curves (fig. 1) showed that each of the four social 
cognitive tests were better at classifying the patients correctly than any of the three executive tests. The AUC was 0.88 for SI-M, 0.86 for RME and 0.79 for both the Emotion Hexagon and the EET. For the Hayling task, the AUC was 0.76, for the FAB it was 0.69 and for the Brixton task it was 0.57 . Due to the small group sizes, we did not calculate cutoff scores with sensitivity and specificity measures.

\section{Discussion}

We compared the performance of 11 patients diagnosed with bvFTD and 10 patients diagnosed with AD. To ensure that our patients were at a relatively early stage of their disease, only patients with a score of 25 or above on the MMSE were included. As another measure of global cognitive function we included the ACE. These measures of global cognitive function showed no significant difference between the two groups. This underscores that such global cognitive measures may be ineffective in differentiating between the two groups at a relatively early stage of the disease when no, or only subtle, neuropsychological impairment may be identifiable. Furthermore, the inclusion of global cognitive measures helps ensure that any differences in the performance on the social cognitive tasks between the two groups were not attributable to differences in overall cognitive function. Along with this, we included three executive tasks, in order to examine whether any differences in the performance on the social cognitive tasks were attributable to differences in executive functioning.

Traditional neuropsychological assessment of patients with bvFTD typically highlights executive impairments, but performance on classic executive tests may show only few abnormalities or even normal performance in the early stages of the disease [25]. Hence, along with the inclusion of the FAB, we also included newer measures of executive functioning, specifically the Hayling and the Brixton tasks. The ability of both these tests to discriminate between bvFTD and $\mathrm{AD}$ patients has previously been investigated, and in one study the Hayling task was found to be the most efficient discriminator among several executive tests examined [37]. In our study, only the Hayling task was able to differentiate between the two groups, and a closer inspection of group performance on the Hayling task reveals that both bvFTD and AD patients actually have very low scores, indicating that this task is quite demanding for both groups. In line with this, impairment on the Hayling task has also been reported in AD patients [38]. Perhaps the low group performance on this task is attributable to different underlying deficits in, for example, memory or executive function, but the overall low scale scores indicate that this task may not have specific clinical utility in differentiating between the two groups. Similar results were seen on the Brixton task where both groups have a relatively high number of errors and a wide range of scores, again indicating that this task may be equally complicated for the two groups. These results showing no or only minor group differences on global and executive tasks indicate that the group differences found on the social cognitive measures are not merely attributable to underlying differences in global or executive functioning. Instead the group differences found on the social cognitive measures may indeed reflect a specific deficit in social cognitive abilities.

By including several social cognitive tasks in this study we have been able to show that these measures were indeed useful in the differentiation between a group of patients with AD and a group of patients with bvFTD at a relatively early stage of the disease (table 2). Our study thus corroborates previous studies showing that various measures of social cognition may indeed show significant differences in the performance between the two patient groups [15, 17-19]. On the EET, a subtest of TASIT, where the subjects had to identify six basic emotions in different video vignettes, the bvFTD group showed significantly lower scores for the total number of correctly identified emotions and the total number of correctly identified negative emotions. There was no difference in the ability of the two groups to correctly identify positive 
Buhl et al.: Clinical Utility of Short Social Cognitive Tests in Early Differentiation of Behavioral Variant Frontotemporal Dementia from Alzheimer's Disease

emotions, which may reflect either a specific deficit in identifying negative emotions in the bvFTD group or an inherently greater difficulty in recognizing negative emotions. On the SI-M of TASIT where the subjects have to understand conversational meanings, determined by paralinguistic cues, the bvFTD group had a poorer performance on both types of sarcastic scenes but performed at the same level when judging the sincere interactions. This confirms that the two groups were equally capable of understanding literal, truthful remarks. Consequently, the significant differences in performance between the two groups cannot be ascribed to any impairment in the ability of the bvFTD group to comprehend the task. Building upon previous studies with similar results [18], the poorer performance of the bvFTD group when judging the sarcastic scenes therefore seems to reflect a specific deficit in understanding the sarcastic 'cues' in the scenes, e.g. facial expressions, bodily gestures or vocal intonation.

On the shorter and more easily administered social cognitive tasks such as the Emotion Hexagon and the RME, the bvFTD group also showed a significantly poorer performance than the AD group. These results are in agreement with previous studies which differentiate between bvFTD and AD patients using emotion recognition tasks $[17,19]$ and theory of mind tasks such as the RME [15].

Since we included various social cognitive tasks, we were able to examine the utility of short and easily administered tasks in comparison with more experimental time-consuming tasks requiring computer access. When evaluating the clinical usefulness of short social cognitive measures in the differentiation between bvFTD and AD, certain issues have to be taken into consideration. The tasks have to be able to differentiate between the two groups, and they have to be easy to administer. In this study, all tasks were well received by our subjects, and the results indicate that both the more experimental social cognitive measures and the shorter social cognitive measures may indeed be capable of detecting early changes in the cognition of patients with bvFTD. Furthermore, these social cognitive tests seem to be able to detect subtle cognitive differences, which are not captured on either global or more specific executive tasks. Although all social cognitive measures showed significant group differences, which could indicate a similar sensitivity to the specific deficits in bvFTD, the results also reveal a great variability in the range of scores presented by the bvFTD group, thus revealing a great deal of heterogeneity within our small sample. This may suggest that certain tasks are better suited for differentiation than others, and our data indicate that the RME and the SI-M may be particularly sensitive to the specific deficits in bvFTD.

Of course detecting specific impairment on various social cognitive tasks does not prove the diagnosis of bvFTD. Nevertheless, very low scores on social cognitive tasks, compared to relatively intact performance on global cognitive measures, may be of clinical relevance in the differentiation between early bvFTD and early AD. Such findings can complement history taking, standardized neuropsychological assessment and clinical observations, and may therefore be valuable in strengthening early diagnosis. Our results suggest that it may indeed be relevant to include these measures of social cognition, but there is still the matter of clinical utility. This necessitates a focus on tasks that are realistic and practical for inclusion in everyday clinical practice. The administration of the shorter social cognitive tasks such as the Emotion Hexagon and the RME requires only static stimulus material consisting of black and white images. In contrast, the EET and the SI-M require computer access and are more timeconsuming. Drawing upon our preliminary results and other studies showing that simple and fast tools are relevant for clinical use $[19,25]$, we therefore propose that short social cognitive tasks such as the RME and the Emotion Hexagon tasks may indeed be used as a valuable supplement in the differential diagnoses of early AD and bvFTD. Due to the small sample size in this study, further investigation is necessary to directly explore the specificity and sensitivity of these tasks. Future studies should therefore seek to include a larger normative sample in order to calculate useful cutoff scores for use in clinical practice. 
Buhl et al:: Clinical Utility of Short Social Cognitive Tests in Early Differentiation of Behavioral Variant Frontotemporal Dementia from Alzheimer's Disease

\section{Acknowledgement}

The Memory Disorders Research Group would like to thank the Ministry of Interior and Health and the Health Insurance Fund for financial support.

\section{References}

1 Rascovsky K, Hodges JR, Knopman D, Mendez MF, Kramer JH, Neuhaus J, van Swieten JC, Seelaar H, Dopper EG, Onyike CU, Hillis AE, Josephs KA, Boeve BF, Kertesz A, Seeley WW, Rankin KP, Johnson JK, Gorno-Tempini ML, Rosen H, Prioleau-Latham CE, Lee A, Kipps CM, Lillo P, Piguet O, Rohrer JD, Rossor MN, Warren JD, Fox NC, Galasko D, Salmon DP, Black SE, Mesulam M, Weintraub S, Dickerson BC, Diehl-Schmid J, Pasquier F, Deramecourt V, Lebert F, Pijnenburg Y, Chow TW, Manes F, Grafman J, Cappa SF, Freedman M, Grossman M, Miller BL: Sensitivity of revised diagnostic criteria for the behavioural variant of frontotemporal dementia. Brain 2011; 134:2456-2477.

-2 McKhann GM, Knopman DS, Chertkow H, Hyman BT, Jack CR Jr, Kawas CH, Klunk WE, Koroshetz WJ, Manly JJ, Mayeux R, Mohs RC, Morris JC, Rossor MN, Scheltens P, Carrillo MC, Thies B, Weintraub S, Phelps CH: The diagnosis of dementia due to Alzheimer's disease: recommendations from the National Institute on AgingAlzheimer's Association workgroups on diagnostic guidelines for Alzheimer's disease. Alzheimers Dement 2011;7:263-269.

-3 Bier JC, Ventura M, Donckels V, Van EE, Claes T, Slama H, Fery P, Vokaer M, Pandolfo M: Is the Addenbrooke's cognitive examination effective to detect frontotemporal dementia? J Neurol 2004;251:428-431.

4 Gregory CA, Orrell M, Sahakian B, Hodges JR: Can frontotemporal dementia and Alzheimer's disease be differentiated using a brief battery of tests? Int J Geriatr Psychiatry 1997;12:375-383.

5 Neary D, Snowden JS, Gustafson L, Passant U, Stuss D, Black S, Freedman M, Kertesz A, Robert PH, Albert M, Boone K, Miller BL, Cummings J, Benson DF: Frontotemporal lobar degeneration: a consensus on clinical diagnostic criteria. Neurology 1998;51:1546-1554.

-6 Torralva T, Roca M, Gleichgerrcht E, Bekinschtein T, Manes F: A neuropsychological battery to detect specific executive and social cognitive impairments in early frontotemporal dementia. Brain 2009;132:1299-1309.

7 Gregory CA, Serra-Mestres J, Hodges JR: Early diagnosis of the frontal variant of frontotemporal dementia: how sensitive are standard neuroimaging and neuropsychologic tests? Neuropsychiatry Neuropsychol Behav Neurol 1999;12:128-135.

$>8$ Lough S, Hodges JR: Measuring and modifying abnormal social cognition in frontal variant frontotemporal dementia. J Psychosom Res 2002;53:639-646.

-9 Lough S, Gregory C, Hodges JR: Dissociation of social cognition and executive function in frontal variant frontotemporal dementia. Neurocase 2001;7:123-130.

10 Graham A, Davies R, Xuereb J, Halliday G, Kril J, Creasey H, Graham K, Hodges J: Pathologically proven frontotemporal dementia presenting with severe amnesia. Brain 2005;128:597-605.

-11 Hornberger M, Piguet 0, Graham AJ, Nestor PJ, Hodges JR: How preserved is episodic memory in behavioral variant frontotemporal dementia? Neurology 2010;74:472-479.

12 Diehl-Schmid J, Pohl C, Ruprecht C, Wagenpfeil S, Foerstl H, Kurz A: The Ekman 60 Faces Test as a diagnostic instrument in frontotemporal dementia. Arch Clin Neuropsychol 2007;22:459-464.

-13 Lough S, Kipps CM, Treise C, Watson P, Blair JR, Hodges JR: Social reasoning, emotion and empathy in frontotemporal dementia. Neuropsychologia 2006;44:950-958.

$>14$ Torralva T, Kipps CM, Hodges JR, Clark L, Bekinschtein T, Roca M, Calcagno ML, Manes F: The relationship between affective decision-making and theory of mind in the frontal variant of fronto-temporal dementia. Neuropsychologia 2007;45:342-349.

-15 Gregory C, Lough S, Stone V, Erzinclioglu S, Martin L, Baron-Cohen S, Hodges JR: Theory of mind in patients with frontal variant frontotemporal dementia and Alzheimer's disease: theoretical and practical implications. Brain 2002;125:752-764.

-16 Keane J, Calder AJ, Hodges JR, Young AW: Face and emotion processing in frontal variant frontotemporal dementia. Neuropsychologia 2002;40:655-665.

17 Fernandez-Duque D, Black SE: Impaired recognition of negative facial emotions in patients with frontotemporal dementia. Neuropsychologia 2005;43:1673-1687.

18 Kipps CM, Nestor PJ, Acosta-Cabronero J, Arnold R, Hodges JR: Understanding social dysfunction in the behavioural variant of frontotemporal dementia: the role of emotion and sarcasm processing. Brain 2009;132: 592-603.

-19 Funkiewiez A, Bertoux M, de Souza LC, Levy R, Dubois B: The SEA (Social Cognition and Emotional Assessment): a clinical neuropsychological tool for early diagnosis of frontal variant of frontotemporal lobar degeneration. Neuropsychology 2012;26:81-90.

20 Bertoux M, Delavest M, de Souza LC, Funkiewiez A, Lepine JP, Fossati P, Dubois B, Sarazin M: Social Cognition and Emotional Assessment differentiates frontotemporal dementia from depression. J Neurol Neurosurg Psychiatry 2012;83:411-416. 
Buhl et al.: Clinical Utility of Short Social Cognitive Tests in Early Differentiation of Behavioral Variant Frontotemporal Dementia from Alzheimer's Disease

21 Seelaar H, Rohrer JD, Pijnenburg YA, Fox NC, van Swieten JC: Clinical, genetic and pathological heterogeneity of frontotemporal dementia: a review. J Neurol Neurosurg Psychiatry 2011;82:476-486.

22 Adenzato M, Cavallo M, Enrici I: Theory of mind ability in the behavioural variant of frontotemporal dementia: an analysis of the neural, cognitive, and social levels. Neuropsychologia 2010;48:2-12.

-23 Shamay-Tsoory SG, Tomer R, Aharon-Peretz J: The neuroanatomical basis of understanding sarcasm and its relationship to social cognition. Neuropsychology 2005;19:288-300.

24 Shamay-Tsoory SG, Tibi-Elhanany Y, Aharon-Peretz J: The ventromedial prefrontal cortex is involved in understanding affective but not cognitive theory of mind stories. Soc Neurosci 2006;1:149-166.

-25 Sarazin M, Dubois B, de Souza LC, Bertoux M: Should the social cognition and emotional assessment replace standard neuropsychological tests for frontotemporal dementia? Expert Rev Neurother 2012;12:633-635.

26 Bertoux M, Funkiewiez A, O'Callaghan C, Dubois B, Hornberger M: Sensitivity and specificity of ventromedial prefrontal cortex tests in behavioral variant frontotemporal dementia. Alzheimers Dement 2012, E-pub ahead of print.

-27 McKhann G, Drachman D, Folstein M, Katzman R, Price D, Stadlan EM: Clinical diagnosis of Alzheimer's disease: report of the NINCDS-ADRDA Work Group under the auspices of Department of Health and Human Services Task Force on Alzheimer's Disease. Neurology 1984;34:939-944.

$\checkmark 28$ Mortensen EL, Gade A: On the relation between demographic variables and neuropsychological test performance. Scand J Psychol 1993;34:305-317.

29 Folstein MF, Folstein SE, McHugh PR: 'Mini-mental state'. A practical method for grading the cognitive state of patients for the clinician. J Psychiatr Res 1975;12:189-198.

-30 Mathuranath PS, Nestor PJ, Berrios GE, Rakowicz W, Hodges JR: A brief cognitive test battery to differentiate Alzheimer's disease and frontotemporal dementia. Neurology 2000;55:1613-1620.

-31 Stokholm J, Vogel A, Johannsen P, Waldemar G: Validation of the Danish Addenbrooke's Cognitive Examination as a screening test in a memory clinic. Dement Geriatr Cogn Disord 2009;27:361-365.

32 Dubois B, Slachevsky A, Litvan I, Pillon B: The FAB: a Frontal Assessment Battery at bedside. Neurology 2000; 55:1621-1626.

33 Burgess PW, Shallice T: The Hayling and Brixton Tests. Bury St. Edmunds, Thames Valley Test Company, 1997.

34 Sprengelmeyer R, Young AW, Calder AJ, Karnat A, Lange H, Homberg V, Perrett DI, Rowland D: Loss of disgust. Perception of faces and emotions in Huntington's disease. Brain 1996;119(Pt 5):1647-1665.

35 Baron-Cohen S, Wheelwright S, Hill J, Raste Y, Plumb I: The 'Reading the Mind in the Eyes' Test revised version: a study with normal adults, and adults with Asperger syndrome or high-functioning autism. J Child Psychol Psychiatry 2001;42:241-251.

36 McDonald S, Flanagan S, Rollins J, Kinch J: TASIT: a new clinical tool for assessing social perception after traumatic brain injury. J Head Trauma Rehabil 2003;18:219-238.

-37 Hornberger M, Savage S, Hsieh S, Mioshi E, Piguet O, Hodges JR: Orbitofrontal dysfunction discriminates behavioral variant frontotemporal dementia from Alzheimer's disease. Dement Geriatr Cogn Disord 2010;30: 547-552.

38 Belleville S, Rouleau N, Van der Linden M: Use of the Hayling task to measure inhibition of prepotent responses in normal aging and Alzheimer's disease. Brain Cogn 2006;62:113-119. 